\title{
Existence Results on General Integrodifferential Evolution Equations in Banach Space
}

\author{
K. Sathiyanathan, T. Nandha Gopal ${ }^{*}$ \\ Department of Mathematics, Sri Ramakrishna Mission Vidyalaya, \\ College of Arts and Science, Coimbatore, India \\ Email: "nandhu792002@yahoo.co.in
}

Received July 2, 2012; revised October 11, 2012; accepted October 18, 2012

\begin{abstract}
In this paper we prove the existence of mild solutions of a general class of nonlinear evolution integrodifferential equation in Banach spaces. Based on the resolvent operator and the Schaefer fixed point theorem, a sufficient condition for the existence of general integrodifferential evolution equations is established.
\end{abstract}

Keywords: Existence; Nonlinear Integrodifferential Evolution Equation; Resolvent Operator; Schaefer’s Fixed Point Theorem

\section{Introduction}

Pazy [1] has discussed the existence and uniqueness of mild, strong and classical solutions of semilinear evolution equations by using semigroup theory. The nonlocal Cauchy problem for the same equation has been studied by Byszewskii [2,3]. Balachandran and Chandrasekaran [4] investigated the nonlocal Cauchy problem for semilinear integrodifferential equation with deviating argument. Balachandran and Park [5] have discussed about the existence of solutions and controllability of nonlinear integrodifferential systems in Banach spaces. Grimmer [6] obtained the representation of solutions of integrodifferential equations by using resolvent operators in a Banach space. Liu [7] discussed the Cauchy problem for integrodifferential evolution equations in abstract spaces and also in [8] he discussed nonautonomous integrodifferential equations.

Lin and Liu [9] studied the nonlocal Cauchy problem for semilinear integrodifferential equations by using resolvent operators. Liu and Ezzinbi [10] investigated nonautonomous integrodifferential equations with nonlocal conditions. Byszewskii and Acka [11] studied the classical solution of nonlinear functional differential equation with time varying delays. There are several papers appeared on the existence of differential and integrodifferential equations in Banach spaces [12,13]. The purpose of this paper is to prove the existence of mild solutions for time varying delay integrodifferential evolution equations with the help of Schaefer's fixed point theorem. The results generalize the results of [14].

${ }^{*}$ Corresponding author.
The paper is organized as follows: In Section 2, we give the necessary definition and gave a description of the idea of the proof of the main results formulated and proved in Section 3. Moreover in Section 3, we prove the existence of solution of general integrodifferential evolution equation with nonlocal condition.

\section{Preliminaries}

Consider the nonlinear delay integrodifferential evolution equation with nonlocal condition of the form

$$
\begin{aligned}
& \frac{\mathrm{d}}{\mathrm{d} t}\left[x(t)-G\left(t, x(t), \int_{0}^{t} g(t, s, x(s)) \mathrm{d} s\right)\right] \\
& =A(t) x(t)+\int_{0}^{t} B(t, s) x(s) \mathrm{d} s \\
& +F\left[t, x(t), \int_{0}^{t} f_{1}(t, s, x(s)) \mathrm{d} s,\right. \\
& \left.\quad \int_{0}^{t} f_{2}(t, s, x(s)) \mathrm{d} s, \cdots, \int_{0}^{t} f_{n}(t, s, x(s)) \mathrm{d} s\right] \\
& t \in J(0)+h(x)=x_{0}
\end{aligned}
$$

where $A(t)$ and $B(t, s)$ are closed linear operators on a Banach space $X$ with dense domain $D(A)$ which is independent of $t$,

$f_{i}: J \times J \times X \rightarrow X, g: J \times J \times X \rightarrow X$,

$h: C(J, X) \rightarrow X, \quad F: J \times X^{n+1} \rightarrow X$ and

$G: J \times X \times X \rightarrow X$ are given functions. Here

$J=[0, T]$.

We shall make the following conditions:

$\left(H_{1}\right) A(t)$ generates a strongly continuous semigroup of evolution operators. 
$\left(\mathrm{H}_{2}\right)$ Suppose $Y$ is a Banach space formed from $D(A)$ with the graph norm. $A(t)$ and $B(t, s)$ are closed operators it follows that $A(t)$ and $B(t, s)$ are in the set of bounded linear operators from $Y$ to $X, B(Y, X)$, for $0 \leq t \leq T$ and $0 \leq s \leq t \leq T$, respectively. $A(t)$ and $B(t, s)$ are continuous on $0 \leq t \leq T$ and $0 \leq s \leq t \leq T$, respectively, into $B(Y, X)$.

Definition 2.1. A resolvent operator for (1) and (2) is a bounded operator valued function $R(t, s) \in B(X)$, $0 \leq s \leq t \leq T$, the space of bounded linear operators on $X$, having the following properties.

(i) $R(t, s)$ is strongly continuous in $s$ and $t . R(t, t)=I$, the identity operator on $X .\|R(t, s)\| \leq M \mathrm{e}^{\beta(t-s)} t, s \in J$ and $M, \beta$ are constants.

(ii) $R(t, s) Y \subset Y, R(t, s)$ is strongly continuous in $\mathrm{s}$ and $t$ on $Y$.

(iii) For $y \in Y, R(t, s) y$ is continuously differentiable in $s$ and $t$, and for $0 \leq s \leq t \leq T$,

$$
\begin{aligned}
\frac{\partial}{\partial t} R(t, s) y= & A(t) R(t, s) y \\
& +\int_{s}^{t} B(t, r) R(r, s) y \mathrm{~d} r, \\
\frac{\partial}{\partial s} R(t, s) y= & -R(t, s) A(s) y \\
& -\int_{s}^{t} R(t, r) B(r, s) y \mathrm{~d} r,
\end{aligned}
$$

with $\frac{\partial}{\partial t} R(t, s) y$ and $\frac{\partial}{\partial s} R(t, s) y$ are strongly continuous on $0 \leq s \leq t \leq T$. Here $R(t, s)$ can be extracted from the evolution operator of the generator $A(t)$. The resolvent operator is similar to the evolution operator for nonautonomous differential equations in Banach spaces.

Definition 2.2. A continuous function $x(t)$ is said to be a mild solution of the nonlocal Cauchy problems (1) and (2), if

$$
\begin{aligned}
x(t)= & R(t, 0)\left[x_{0}-h(x)\right]-R(t, 0)[G(0, x(0), 0)]+G\left(t, x(t), \int_{0}^{t} g(t, s, x(s)) \mathrm{d} s\right) \\
& +\int_{0}^{t} R(t, s) F\left(s, x(s), \int_{0}^{s} f_{1}(s, \tau, x(\tau)) \mathrm{d} \tau, \cdots, \int_{0}^{s} f_{n}(s, \tau, x(\tau)) \mathrm{d} \tau\right) \mathrm{d} s \\
& -\int_{0}^{t} R(t, s) A(s) G\left(s, x(s), \int_{0}^{s} g(s, \tau, x(\tau)) \mathrm{d} \tau\right) \mathrm{d} s \\
& -\int_{0}^{t}\left[\int_{s}^{t} R(t, \tau) B(\tau, s) G\left(\tau, x(\tau), \int_{0}^{\tau} g(\tau, \zeta, x(\zeta)) \mathrm{d} \zeta\right) \mathrm{d} \tau\right] \mathrm{d} s
\end{aligned}
$$

is satisfied.

Schaefer's Theorem [15]. Let $E$ be a normed linear space. Let $F: E \rightarrow E$ be a completely continuous operator, that is, it is continuous and the image of any bounded set is contained in a compact set and let

$$
\zeta(F)=\{x \in E: x=\lambda F x \text { for some } 0<\lambda<1\}
$$

Then either $\zeta(F)$ is unbounded or $\mathrm{F}$ has a fixed point.

Assume that the following conditions hold:

$\left(H_{3}\right)$ There exists a resolvent operator $R(t, s)$ which is compact and continuous in the uniform operator topology for $t>s$. Further, there exists a constant $M_{1}>0$ such that

$$
\|R(t, s)\| \leq M_{1} .
$$

$\left(H_{4}\right)$ The function $h: C(J, X) \rightarrow X$ is continuous and there exists a constant $M_{2}>0$ such that $\|h(x)\| \leq M_{2}$ for any $x \in X$.

$\left(H_{5}\right)$ For each $t \in J$, the function

$F(t, \cdot): X^{n+1} \rightarrow X$ is continuous and for each $\left(x_{0}, x_{1}, \cdots, x_{n}\right) \in X^{n+1}$ the function $F\left(., x_{0}, x_{1}, \cdots, x_{n}\right): J \rightarrow X$ is strongly measurable.

$\left(H_{6}\right)$ There exists an integrable function $m_{i}: J \rightarrow[0, \infty)$ such that

$$
\left\|f_{i}(t, s, x)\right\| \leq m_{i}(t, s) \Omega_{i}(\|x\|) \text {, for any } t, s \in J, x \in X,
$$

for any $t \in J, x \in X$. where $\Omega_{i}:[0, \infty) \rightarrow(0, \infty)$ is a continuous nondecreasing function.

$\left(H_{7}\right)$ Ther exists an integrable function $m_{0}: J \times J \rightarrow[0, \infty)$ such that

$$
\|g(t, s, x)\| \leq m_{0}(t, s) \Omega_{0}(\|x\|) \text {, for any } t, s \in J, x \in X \text {, }
$$

where $\Omega_{0}:[0, \infty) \rightarrow[0, \infty)$ is a continuous nondecreasing function.

$\left(H_{8}\right)$ The function $G: C(J, X) \rightarrow X$ is completely continuous and there exists a constant $0 \leq c_{1} \leq 1$ such that

$$
\|G(t, x(t), y(t))\| \leq c_{1}[\|x(t)\|+\|y(t)\|] \text {, for any } t \in J
$$

and is equicontinuous in $(J, X)$

$\left(H_{9}\right)$ The function $G: C(J, X) \rightarrow X$ is completely continuous and there exists a constant $0 \leq c_{2} \leq 1$ such that

$$
\|F(t, x(t), y(t))\| \leq c_{2}[\|x(t)\|+\|y(t)\|] \text {, for any } t \in J
$$

and is equicontinuous in $(J, X)$

$\left(H_{10}\right)$ There are function

$H_{1}(),. H_{2}(),. \mu_{1}(),. \mu_{2}():. J \rightarrow[0, \infty]$ such that

$$
\begin{gathered}
\|A(t) R(t, s)\| \leq H_{1}(t) \mu_{1}(s) \\
\|B(t, s) R(t, s)\| \leq H_{2}(t) \mu_{2}(s) .
\end{gathered}
$$


$\left(H_{11}\right)$ The function

$$
\begin{aligned}
& \hat{m}(t)=\max \left\{\frac{1}{1-c_{1}} M_{1} c_{2}, \frac{M_{1} c_{2}}{1-c_{1}} \sum_{i=1}^{n} m_{i}(t, s),\right. \\
& \frac{c_{1}}{1-c_{1}} m_{0}(t, s), \frac{c_{1}}{1-c_{1}} H_{1}(t) \mu_{1}(t), \\
& \frac{c_{1}}{1-c_{1}} H_{1}(t) \mu_{1}(t) m_{0}(t, s), \\
& \frac{c_{1}}{1-c_{1}} \int_{s}^{t} H_{2}(s) \mu_{2}(s) \mathrm{d} s, \\
&\left.\frac{c_{1}}{1-c_{1}} \int_{s}^{t} \int_{0}^{s} H_{1}(t) \mu_{1}(t) m_{0}(t, s) \mathrm{d} t \mathrm{~d} s\right\} \\
& \int_{0}^{T} \hat{m}(s) \mathrm{d} s<\int_{c}^{\infty} \frac{\mathrm{d} s}{3 s+2 \Omega_{0}(s)+\sum_{i=1}^{n} \Omega_{i}(s)}
\end{aligned}
$$

where $c=\frac{1}{1-c_{1}} M_{1}\left[\left\|x_{0}\right\|+M_{2}+G(0, x(0), 0)\right]$

\section{Existence of Mild Solutions}

The main result is as follows.

Theorem 3.1. If the assumptions $\left(H_{1}\right)-\left(H_{11}\right)$ are satisfied then the problems (1) and (2) has a mild solution on J.

Proof: Consider the Banach space $Z=C(J, X)$. We establish the existence of a mild solution of the problems (1) and (2) by applying the Schaefer's fixed point theorem.

First we obtain a priori bounds for the operator equation

$$
x(t)=\lambda \Phi x(t), \quad 0<\lambda<1,
$$

where $\Phi: Z \rightarrow Z$ is defined as

$$
\begin{aligned}
(\Phi x)(t)= & R(t, 0)\left[x_{0}-h(x)-G(0, x(0), 0)\right]+\int_{0}^{t} R(t, s) F\left(s, x(s), \int_{0}^{s} f_{1}(s, \tau, x(\tau)) \mathrm{d} \tau, \cdots, \int_{0}^{s} f_{n}(s, \tau, x(\tau)) \mathrm{d} \tau\right) \mathrm{d} s \\
& +G\left(t, x(t), \int_{0}^{t} g(t, s, x(s)) \mathrm{d} s\right)-\int_{0}^{t} R(t, s) A(s) G\left(s, x(s), \int_{0}^{s} g(s, \tau, x(\tau)) \mathrm{d} \tau\right) \mathrm{d} s \\
& -\int_{0}^{t}\left(\int_{s}^{t} R(t, \tau) B(\tau, s) G\left(\tau, x(\tau), \int_{0}^{\tau} g(\tau, \zeta, x(\zeta)) \mathrm{d} \zeta\right) \mathrm{d} \tau\right) \mathrm{d} s
\end{aligned}
$$

Then froms (3) and (4) we have

$$
\begin{aligned}
&\|x(t)\| \leq M_{1}\left[\left\|x_{0}\right\|+M_{2}+G(0, x(0), 0)\right]+M_{1} c_{2} \int_{0}^{t}\left[\|x(t)\|+\sum_{i=1}^{n} m_{i}(t, s) \Omega_{i}(\|x(s)\|)\right] \mathrm{d} s \\
&+ c_{1}\left[\|x(t)\|+\int_{0}^{t} m_{0}(t, s) \Omega_{0}(\|x(s)\|) \mathrm{d} s\right]+c_{1} \int_{0}^{t} H_{1}(s) \mu_{1}(s)\left[\|x(s)\|+\int_{0}^{s} m_{0}(s, \tau) \Omega_{0}(\|x(\tau)\|) \mathrm{d} \tau\right] \mathrm{d} s \\
&+c_{1} \int_{0}^{t}\left[\int_{s}^{t} H_{2}(\tau) \mu_{2}(\tau)\left[\|x(s)\|+\int_{0}^{\tau} m_{0}(\tau, \zeta) \Omega_{0}(\|x(\zeta)\|) \mathrm{d} \zeta\right] \mathrm{d} \tau\right] \mathrm{d} s \\
&\left(1-c_{1}\right)\|x(t)\| \leq M_{1}\left[\left\|x_{0}\right\|+M_{2}+G(0, x(0), 0)\right]+c_{1} \int_{0}^{t} m_{0}(t, s) \Omega_{0}(\|x(s)\|) \mathrm{d} s \\
&+M_{1} c_{2} \int_{0}^{t}\left[\|x(t)\|+\sum_{i=1}^{n} m_{i}(t, s) \Omega_{i}(\|x(s)\|)\right] \mathrm{d} s \\
&+c_{1} \int_{0}^{t} H_{1}(s) \mu_{1}(s)\left[\|x(s)\|+\int_{0}^{s} m_{0}(s, \tau) \Omega_{0}(\|x(\tau)\|) \mathrm{d} \tau\right] \mathrm{d} s \\
&+c_{1} \int_{0}^{t}\left[\int_{s}^{t} H_{2}(\tau) \mu_{2}(\tau)\left[\|x(s)\|+\int_{0}^{\tau} m_{0}(\tau, \zeta) \Omega_{0}(\|x(\zeta)\|) \mathrm{d} \zeta\right] \mathrm{d} \tau\right] \mathrm{d} s \\
& 1 \\
&\|x(t)\| \leq \frac{1}{\left(1-c_{1}\right)}\left\{M_{1}\left[\left\|x_{0}\right\|+M_{2}+G(0, x(0), 0)\right]+c_{1} \int_{0}^{t} m_{0}(t, s) \Omega_{0}(\|x(s)\|) \mathrm{d} s\right. \\
&+ M_{1} c_{2} \int_{0}^{t}\left[\|x(t)\|+\sum_{i=1}^{n} m_{i}(t, s) \Omega_{i}(\|x(s)\|)\right] \mathrm{d} s \\
&+ c_{1} \int_{0}^{t} H_{1}(s) \mu_{1}(s)\left[\|x(s)\|+\int_{0}^{s} m_{0}(s, \tau) \Omega_{0}(\|x(\tau)\|) \mathrm{d} \tau\right] \mathrm{d} s \\
&+\left.c_{1} \int_{0}^{t}\left[\int_{s}^{t} H_{2}(\tau) \mu_{2}(\tau)\left[\|x(s)\|+\int_{0}^{\tau} m_{0}(\tau, \zeta) \Omega_{0}(\|x(\zeta)\|) \mathrm{d} \zeta\right] \mathrm{d} \tau\right] \mathrm{d} s\right\}
\end{aligned}
$$


Denoting the right hand side of the above inequality as $v(t)$. Then $\|x(t)\| \leq v(t)$ and

$$
\begin{aligned}
& v(0)=c=\frac{1}{1-c_{1}} M_{1}\left[\left\|x_{0}\right\|+M_{2}+G(0, x(0), 0)\right] . \\
& v^{\prime}(t)=\frac{1}{1-c_{1}}\left\{c_{1} m_{0}(t, s) \Omega_{0}(v(t))\right. \\
& +M_{1} c_{2}\left[v(t)+\sum_{i=1}^{n} m_{i}(t, s) \Omega_{i}(v(t))\right] \\
& +c_{1} H_{1}(t) \mu_{1}(t)\left[v(t)+\int_{0}^{t} m_{0}(t, s) \Omega_{0}(v(s)) \mathrm{d} s\right] \\
& \left.+c_{1}\left[\int_{s}^{t} H_{2}(s) \mu_{2}(s)\left[v(t)+\int_{0}^{s} m_{0}(s, \tau) \Omega_{0}(v(\tau)) \mathrm{d} \tau\right] \mathrm{d} s\right]\right\} \\
& \leq \hat{m}(t)\left[\Omega_{0}(v(t))+v(t)+\sum_{i=1}^{n} \Omega_{i}(v(t))\right],
\end{aligned}
$$

This implies

$$
\begin{aligned}
& \int_{v(0)}^{v(t)} \frac{\mathrm{d} s}{3 s+\sum_{i=1}^{n} \Omega_{i}(s)+2 \Omega_{0}(s)} \leq \int_{0}^{T} \hat{m}(s) \mathrm{d} s \\
& <\int_{c}^{\infty} \frac{\mathrm{d} s}{3 s+\sum_{i=1}^{n} \Omega_{i}(s)+2 \Omega_{0}(s)}, 0 \leq t \leq T .
\end{aligned}
$$

where $c=\frac{1}{1-c_{1}} M_{1}\left[\left\|x_{0}\right\|+M_{2}+G(0, x(0), 0)\right]$

Inequality (5) implies that there is a constant $K$ such that $v(t) \leq K, t \in J$ and hence we have

$\|x\|=\sup \{|x(t)|: t \in J\} \leq K$, where $K$ depends only on $T$ and on the functions $\hat{m}, \Omega_{0}$ and $\Omega_{i}$.

We shall now prove that the operator $\Phi: Z \rightarrow Z$ is a completely continuous operator. Let

$B_{k}=\{x \in Z:\|x\| \leq k\}$ for some $k \geq 1$. We first show that $\Phi$ maps $B_{k}$ into an equicontinuous family.

Let $x \in B_{k}$ and $t_{1}, t_{2} \in[0, T]$. Then if $0<t_{1}<t_{2}<T$

$$
\begin{aligned}
& \left\|(\Phi x)\left(t_{1}\right)-(\Phi x)\left(t_{2}\right)\right\| \leq\left\|\left(R\left(t_{1}, 0\right)-R\left(t_{2}, 0\right)\right)\left[x_{0}-h(x)-G(0, x(0), 0)\right]\right\| \\
& +\left\|\int_{0}^{t_{1}}\left[\left[R\left(t_{1}, s\right)-R\left(t_{2}, s\right)\right] F\left(s, x(s), \int_{0}^{s} f_{1}(s, \tau, x(\tau)) \mathrm{d} \tau, \cdots, \int_{0}^{s} f_{n}(s, \tau, x(\tau)) \mathrm{d} \tau\right)\right] \mathrm{d} s\right\| \\
& +\left\|G\left(t_{1}, x\left(t_{1}\right), \int_{0}^{t_{1}} g\left(t_{1}, s, x(s)\right) \mathrm{d} s\right)-G\left(t_{2}, x\left(t_{2}\right), \int_{0}^{t_{2}} g\left(t_{2}, s, x(s)\right) \mathrm{d} s\right)\right\| \\
& +\left\|\int_{0}^{t_{1}}\left[\left(R\left(t_{1}, s\right)-R\left(t_{2}, s\right)\right) A(s) G\left(s, x(s), \int_{0}^{s} g(s, \tau, x(\tau)) \mathrm{d} \tau\right)\right] \mathrm{d} s\right\| \\
& +\left\|\int_{0}^{\mathbf{t}_{1}}\left[\int_{\mathbf{s}}^{\mathbf{t}_{1}}\left(\mathbf{R}\left(\mathbf{t}_{1}, \tau\right)-\mathbf{R}\left(\mathbf{t}_{2}, \tau\right)\right) \mathbf{B}(\tau, \mathbf{s}) \mathbf{G}\left(\mathbf{t}, \mathbf{x}(\tau), \int_{0}^{\tau} \mathbf{g}(\tau, \zeta, \mathbf{x}(\zeta)) \mathbf{d} \zeta\right) \mathbf{d} \tau\right] \mathbf{d s}\right\| \\
& +\left\|\int_{t_{1}}^{t_{2}}\left[R\left(t_{2}, s\right) F\left(s, x(s), \int_{0}^{s} f_{1}(s, \tau, x(\tau)) \mathrm{d} \tau, \cdots, \int_{0}^{s} f_{n}(s, \tau, x(\tau)) \mathrm{d} \tau\right)\right] \mathrm{d} s\right\| \\
& +\| \int_{t_{1}}^{t_{2}}\left[R\left(t_{2}, s\right) A(s) G\left(s, x(s), \int_{0}^{s} g(s, \tau, x(\tau)) \mathrm{d} \tau\right] \mathrm{d} s \|\right. \\
& +\left\|\int_{\mathbf{t}_{1}}^{\mathbf{t}_{2}}\left[\int_{\mathbf{s}}^{\mathbf{t}_{2}}\left(\mathbf{R}\left(\mathbf{t}_{2}, \mathbf{s}\right) \mathbf{B}(\tau, \mathbf{s}) \mathbf{G}\left(\mathbf{t}, \mathbf{x}(\tau), \int_{0}^{\tau} \mathbf{g}(\tau, \zeta, \mathbf{x}(\zeta)) \mathbf{d} \zeta\right)\right) \mathbf{d} \tau\right] \mathbf{d s}\right\| \\
& \leq\left\|\left(R\left(t_{1}, 0\right)-R\left(t_{2}, 0\right)\right)\left[x_{0}-h(x)-G(0, x(0), 0)\right]\right\| \\
& +\left\|\int_{0}^{t_{1}}\left[\left[R\left(t_{1}, s\right)-R\left(t_{2}, s\right)\right] F\left(s, x(s), \int_{0}^{s} f_{1}(s, \tau, x(\tau)) \mathrm{d} \tau, \cdots, \int_{0}^{s} f_{n}(s, \tau, x(\tau)) \mathrm{d} \tau\right)\right] \mathrm{d} s\right\| \\
& +\left\|G\left(t_{1}, x\left(t_{1}\right), \int_{0}^{t_{1}} g\left(t_{1}, s, x(s)\right) \mathrm{d} s\right)-G\left(t_{2}, x\left(t_{2}\right), \int_{0}^{t_{2}} g\left(t_{2}, s, x(s)\right) \mathrm{d} s\right)\right\| \\
& +\left\|\int_{0}^{t_{1}}\left[\left(R\left(t_{1}, s\right)-R\left(t_{2}, s\right)\right) A(s) G\left(s, x(s), \int_{0}^{s} g(s, \tau, x(\tau)) \mathrm{d} \tau\right)\right] \mathrm{d} s\right\| \\
& +\left\|\int_{0}^{\mathrm{t}_{1}}\left[\int_{\mathrm{s}}^{\mathrm{t}_{1}}\left(\mathbf{R}\left(\mathbf{t}_{1}, \tau\right)-\mathbf{R}\left(\mathbf{t}_{2}, \tau\right)\right) \mathbf{B}(\tau, \mathrm{s}) \mathbf{G}\left(\tau, \mathrm{x}(\tau), \int_{0}^{\tau} \mathrm{g}(\tau, \zeta, \mathrm{x}(\zeta)) \mathbf{d} \zeta\right) \mathbf{d} \tau\right] \mathbf{d s}\right\| \\
& +M_{1} c_{2} \int_{t_{1}}^{t_{2}} \sum_{i=1}^{n}\left[k+m_{i}(t, s) \Omega_{i}(k)\right] \mathrm{d} s-M_{1} c_{1} \int_{t_{1}}^{t_{2}} H_{1}(s) \mu_{1}(s)\left[k+\int_{0}^{s} m_{0}(s, \tau) \Omega_{0}(k) \mathrm{d} \tau\right] \mathrm{d} s \\
& +\mathbf{M}_{1} \mathbf{c}_{1} \int_{t_{1}}^{t_{2}}\left[\int_{s}^{t_{2}} \mathbf{H}_{1}(\tau) \mu_{1}(\tau)\left[\mathrm{k}+\int_{0}^{\mathrm{s}} \mathrm{m}_{0}(\mathrm{~s}, \zeta) \Omega_{0}(\mathrm{k}) \mathbf{d} \zeta\right] \mathbf{d} \tau\right] \mathbf{d} \mathbf{s}
\end{aligned}
$$


The right hand side is independent of $x \in B_{k}$ and tends to zero as $t_{2}-t_{1} \rightarrow 0$, since $\mathrm{f}$ is completely continuous and by $\left(H_{3}\right), R(t, s)$ for $t>s$ is continuous in the uniform operator topology. Thus $\Phi$ maps $B_{k}$ into an equicontinuous family of functions.

It is easy to see that $\Phi B_{k}$ is uniformly bounded. Next, we show $\overline{\Phi B_{k}}$ is compact. Since we have shown $\Phi B_{k}$ is equicontinuous collection, by the Arzela-Ascoli theorem it suffices to show that $\Phi$ maps $B_{k}$ into a precompact set in $X$.

Let $0<t \leq T$ be fixed and let $\epsilon$ be a real number satisfying $0<\epsilon<t$. For $x \in B_{k}$, we define

$$
\begin{aligned}
\left(\Phi_{\epsilon} x\right)(t)= & R(t, 0)\left[x_{0}-h(x)-G(0, x(0), 0)\right]+G\left(t, x(t), \int_{0}^{t-\epsilon} g(t, s, x(s)) \mathrm{d} s\right) \\
& +\int_{0}^{t-\epsilon} R(t, s) F\left(s, x(s), \int_{0}^{s} f_{1}(s, \tau, x(\tau)) \mathrm{d} \tau, \cdots, \int_{0}^{s} f_{n}(s, \tau, x(\tau)) \mathrm{d} \tau\right) \mathrm{d} s \\
& -\int_{0}^{t-\epsilon}\left[\int_{s}^{t-\epsilon} R(t, \tau) B(\tau, s) G\left(\tau, x(\tau), \int_{0}^{\tau} g(\tau, \zeta, x(\zeta)) \mathrm{d} \zeta\right) \mathrm{d} \tau\right] \mathrm{d} s \\
& -\int_{0}^{t-\epsilon}\left[R(t, s) A(s) G\left(s, x(s), \int_{0}^{s} g(s, \tau, x(\tau)) \mathrm{d} \tau\right)\right] \mathrm{d} s
\end{aligned}
$$

Since $R(t, s)$ is a compact operator, the set $Y_{\epsilon}(t)=\left\{\left(\Phi_{\epsilon} x\right)(t): x \in B_{k}\right\}$ is precompact in $X$ for every $\epsilon, 0<\epsilon<t$. Moreover, for every $x \in B_{k}$ we have

$$
\begin{aligned}
& \left\|(\Phi x)(t)-\left(\Phi_{\epsilon} x\right)(t)\right\| \leq \int_{t-\epsilon}^{t} R(t, s) F\left(s, x(s), \int_{0}^{s} f_{1}(s, \tau, x(\tau)) \mathrm{d} \tau, \cdots, \int_{0}^{s} f_{n}(s, \tau, x(\tau)) \mathrm{d} \tau\right) \mathrm{d} s \\
& +\int_{\mathbf{t}-\varepsilon}^{\mathbf{t}}\left[\int_{\mathrm{s}}^{\mathbf{t}-\epsilon} \mathbf{R}(\mathbf{t}, \tau) \mathbf{B}(\tau, \mathrm{s})\left[\mathbf{G}\left(\tau, \mathrm{x}(\tau), \int_{0}^{\tau} \mathrm{g}(\mathrm{s}, \zeta, \mathrm{x}(\zeta)) \mathbf{d} \zeta\right)\right] \mathbf{d} \tau\right] \mathbf{d s} \\
& +\int_{t-\varepsilon}^{t}\left[R(t, s) A(s) G\left(s, x(s), \int_{0}^{s} g(s, \tau, x(\tau)) \mathrm{d} \tau\right)\right] \mathrm{d} s \\
& \leq M_{1} c_{2} \int_{t-\epsilon}^{t} \sum_{i=1}^{n}\left[k+m_{i}(t, s) \Omega_{i}(k) \mathrm{d} s\right] \\
& +M_{1} c_{1} \int_{t-\epsilon}^{t} H_{1}(s) \mu_{1}(s)\left[k+\int_{0}^{s} m_{0}(s, \tau) \Omega_{0}(k) \mathrm{d} \tau\right] \mathrm{d} s \\
& +M_{1} c_{1} \int_{\mathbf{t}-\epsilon}^{\mathbf{t}}\left[\int_{s}^{\mathbf{t}-\epsilon} H_{1}(\tau) \mu_{1}(\tau)\left[\mathbf{k}+\int_{0}^{\tau} \mathbf{m}_{0}(\tau, \zeta) \Omega_{0}(\mathbf{k}) \mathbf{d} \zeta\right] \mathbf{d} \tau\right] \mathbf{d s}
\end{aligned}
$$

Therefore there are precompact sets arbitrarily close to the set $\left\{(\Phi x)(t): x \in B_{k}\right\}$.

Hence, the set $\left\{(\Phi x)(t): x \in B_{k}\right\}$ is precompact in $X$.
It remains to show that $\Phi: Z \rightarrow Z$ is continuous. Let $\left\{x_{n}\right\}_{0}^{\infty} \subseteq Z$ with $x_{n} \rightarrow x$ in $Z$. Then there is an integer q such that $\left\|x_{n}(t)\right\| \leq q$ for all $n$ and $t \in J$, so $x_{n} \in B_{q}$ and $x \in B_{q}$. By $\left(H_{4}\right)$,

$$
\begin{aligned}
& F\left(t, x_{n}(t), \int_{0}^{t} f_{1}\left(t, s, x_{n}(s)\right) \mathrm{d} s, \int_{0}^{t} f_{2}\left(t, s, x_{n}(s)\right) \mathrm{d} s, \cdots, \int_{0}^{t} f_{n}\left(t, s, x_{n}(s)\right) \mathrm{d} s\right) \\
& \rightarrow F\left(t, x(t), \int_{0}^{t} f_{1}(t, s, x(s)) \mathrm{d} s, \int_{0}^{t} f_{2}(t, s, x(s)) \mathrm{d} s, \cdots, \int_{0}^{t} f_{n}(t, s, x(s)) \mathrm{d} s\right)
\end{aligned}
$$

for each $t \in J$ and since

$$
\begin{aligned}
& \| F\left(t, x_{n}(t), \int_{0}^{t} f_{1}\left(t, \tau, x_{n}(\tau)\right) \mathrm{d} \tau, \int_{0}^{t} f_{2}\left(t, \tau, x_{n}(\tau)\right) \mathrm{d} \tau, \cdots, \int_{0}^{t} f_{n}\left(t, \tau, x_{n}(\tau)\right) \mathrm{d} \tau\right) \\
& -F\left(t, x(t), \int_{0}^{t} f_{1}(t, \tau, x(\tau)) \mathrm{d} \tau, \int_{0}^{t} f_{2}(t, \tau, x(\tau)) \mathrm{d} \tau, \cdots, \int_{0}^{t} f_{n}(t, \tau, x(\tau)) \mathrm{d} \tau\right) \| \\
& \leq 2 c_{2} m_{i}(t, \tau) \Omega_{i}(q)\left\|g\left(t, \tau, x_{n}(\tau)\right)-g(t, \tau, x(\tau))\right\| \leq 2 m_{0}(t, \tau) \Omega_{0}(q) \\
& \left\|A(\tau) R(t, \tau) G\left(\tau, x_{n}(\tau), \int_{0}^{\tau} g\left(t, \tau, x_{n}(\tau)\right) \mathrm{d} \tau\right)-A(\tau) R(t, \tau) G\left(\tau, x(\tau), \int_{0}^{\tau} g(t, \tau, x(\tau)) \mathrm{d} \tau\right)\right\| \\
& \leq 2 c_{1} \mu_{1}(t) H_{1}(t) q
\end{aligned}
$$

and 


$$
\begin{aligned}
& \left\|B(t, \tau) R(t, \tau) G\left(\tau, x_{n}(\tau), \int_{0}^{\tau} g\left(t, \tau, x_{n}(\tau)\right) \mathrm{d} \tau\right)-B(t, \tau) R(t, \tau) G\left(\tau, x(\tau), \int_{0}^{\tau} g(t, \tau, x(\tau)) \mathrm{d} \tau\right)\right\| \\
& \leq 2 c_{1} \mu_{2}(t) H_{2}(t) q \quad 0 \leq c_{1} \leq 1,0 \leq c_{2} \leq 1 .
\end{aligned}
$$

we have by dominated convergence theorem

$$
\begin{aligned}
& \left\|\Phi x_{n}-\Phi_{x}\right\| \leq \int_{0}^{t} \| R(t, s)\left[F\left(t, x_{n}(t), \int_{0}^{t} f_{1}\left(t, \tau, x_{n}(\tau)\right) \mathrm{d} \tau, \cdots, \int_{0}^{t} f_{n}\left(t, \tau, x_{n}(\tau)\right) \mathrm{d} \tau\right)\right. \\
& \left.-F\left(t, x(t), \int_{0}^{t} f_{1}(t, \tau, x(\tau)) \mathrm{d} \tau, \cdots, \int_{0}^{t} f_{n}(t, \tau, x(\tau)) \mathrm{d} \tau\right)\right] \| \mathrm{d} s \\
& +\int_{0}^{t}\left\|R(t, s)\left[g\left(t, \tau, x_{n}(\tau)\right)-g(t, \tau, x(\tau))\right]\right\| \mathrm{d} s \\
& +\int_{0}^{t}\|R(t, s)\|\left\|A(\tau) R(t, \tau) G\left(\tau, x_{n}(\tau), \int_{0}^{\tau} g\left(t, \tau, x_{n}(\tau)\right) \mathrm{d} \tau\right)-A(\tau) R(t, \tau) G\left(\tau, x(\tau), \int_{0}^{\tau} g(t, \tau, x(\tau)) \mathrm{d} \tau\right)\right\| \mathrm{d} s \\
& +\int_{0}^{t}\left[\int_{0}^{s}\left\|B(t, \tau) R(t, \tau) G\left(\tau, x_{n}(\tau), \int_{0}^{\tau} g\left(t, \zeta, x_{n}(\zeta)\right) \mathrm{d} \zeta\right)-B(t, \tau) R(t, \tau) G\left(\tau, x(\tau), \int_{0}^{\tau} g(t, \zeta, x(\zeta)) \mathrm{d} \zeta\right)\right\| \mathrm{d} \tau\right] \mathrm{d} s \rightarrow 0 \\
& \text { as } n \rightarrow \infty
\end{aligned}
$$

Thus $\Phi$ is continuous. This completes the proof that $\Phi$ is completely continuous.

Finally the set $\zeta(\Phi)=\{x \in Z: x=\lambda \Phi x, \lambda \in(0,1)\}$ is bounded, as we proved in the first step. Consequently, by Schaefer's theorem, the operator $\Phi$ has a fixed point in $Z$. This means that any fixed point of $\Phi$ is a mild solution of (1) and (2) on $J$ satisfying $(\Phi x)(t)=x(t)$.

\section{REFERENCES}

[1] A. Pazy, "Semigroups of Linear Operators and Applications to Partial Differential Equations," Springer-Verlag, New York, 1933.

[2] L. Byszewski, "Theorems about the Existences and Uniqueness of a Solutions of a Semilinear Evolution Nonlocal Cauchy Problem,” Journal of Mathematical Analysis and Application, Vol. 162, No. 2, 1991, pp. 496-505. doi:10.1016/0022-247X(91)90164-U

[3] L. Byszewski, "Applications of Properties of the RightHand Sides of Evolution Equations to an Investigation of Nonlocal Evolution Problems,” Nonlinear Analysis, Vol. 33, No. 5, 1998, pp. 413-426. doi:10.1016/S0362-546X(97)00594-4

[4] K. Balachandran and M. Chandrasekaran, "The Nonlocal Cauchy Problem for Semilinear Integrodifferential Equation with Devating Argument," Proceedings of the Edinburgh Mathematical Society, Vol. 44, No. 1, 2001, pp. 6370. doi:10.1017/S0013091598001060

[5] K. Balachandran and J. Y. Park, "Existence of Solutions and Controllability of Nonlinear Integrodifferential Systems in Banach Spaces," Mathematical Problems in Engineering, Vol. 2003, No. 2, 2003, pp. 65-79. doi:10.1155/S1024123X03201022

[6] R. Gimmer, "Resolvent Operators for Integral Equations in a Banach Space," Transactions of the American Mathematical Society, Vol. 273, 1982, pp. 333-349.

$$
\text { doi:10.1090/S0002-9947-1982-0664046-4 }
$$

[7] J. H. Liu, "Resolvent Operators and Weak Solutions of Integrodifferential Equations," Differential and Integral Equations, Vol. 7, 1994, pp. 523-534.

[8] J. H. Liu, "Integrodifferential Equations with Nonautonomous Operators," Dynamic Systems and Applications, Vol. 7, 1998, pp. 427-440.

[9] Y. Lin and J. H. Liu, "Semilinear Integrodifferential Equations with Nonlocal Cauchy Problem," Nonlinear Analysis; Theory, Methods and Applications, Vol. 26, 1996, pp. 1023-1033.

[10] J. H. Liu and K. Ezzinbi, "Non-Autonomous Integrodifferential Equations with Nonlocal Conditions," Journal of Integral Equations and Applications, Vol. 15, No. 1, 2003, pp. 79-93. doi:10.1216/jiea/1181074946

[11] L. Byszewski and H. Acka, "Existence of Solutions of a Semilinear Functional Differential Evolution Nonlocal Problem," Nonlinear Analysis, Vol. 34, No. 1, 1998, pp. 65-72. doi:10.1016/S0362-546X(97)00693-7

[12] K. Balachandran, J. H. Kim and A. Leelamani, "Existence Results for Nonlinear Abstract Neutral Differential Equations with Time Varying Delays," Applied Mathematics E-Notes, Vol. 6, 2006, pp. 186-193.

[13] H. M. Eduardo, H. R. Henriquez and J. P. C. dos Santos, "Existence Results for Abstract Partial Neutral Integrodifferential Equation with Unbounded Delay,” Electronic Journal of Qualitative Theory of Differential Equations, Vol. 29, 2009, pp. 1-23.

[14] K. Balachandran and R. Ravikumar, "Existence of Solutions of Integrodifferential Evolution Equations with Time Varying Delays,” Applied Mathematics E-Notes, Vol. 7, 2007, pp. 1-8.

[15] H. Schaefer, "Uber Die Methods der a Priori Schranken," Mathematische Annalem, Vol. 129, No. 1, 1955, pp. 415416. doi:10.1007/BF01362380 